\title{
BIANCA-LIVIA BARTOȘ
}

Université Babeș-Bolyai de Cluj-Napoca

\section{Hervé Bazin et le baptême de l'air - gratification du dieu Éther}

\author{
Partir, c'est mourir un peu, \\ C'est mourir à ce qu'on aime : \\ On laisse un peu de soi-même \\ En toute heure et dans tout lieu. \\ E. Haraucourt ${ }^{1}$
}

T a seconde moitié du XXe siècle ouvre les portes d'une nouvelle vision romanesque dans l'univers des belles lettres. Parmi les grands auteurs qui ont contribué à cette révolution du genre littéraire se trouve aussi Hervé Bazin, écrivain angevin qui se déclare insoumis face à la tradition et précurseur du roman moderne. Le but de cette recherche est de remettre en discussion son œuvre passionnante, pour ainsi mettre en évidence le concept de voyage dans sa vision : préférant l'univers tellurique à la rivière, Hervé Bazin transforme le topos de ses romans dans un cadre privilégié pour l'écriture. Et puisque son écriture est innovative, elle réclame un renouvellement perpétuel de l'espace après chaque roman publié : c'est ainsi que le personnage bazinien se définit par l'esprit de la bougeotte, étant un passionné de l'Éther, cet espace vide qu'il remplit avec son souffle créateur et par le dynamisme de son être.

Selon Hésiode, au commencement était le Chaos. De son union avec Gaïa naquirent Érèbe et la Nuit qui, en

${ }^{1}$ E. Haraucourt, "Rondel de l'adieu », Seul, Paris, Bibliothèque Charpentier, 1891, p. 12. 
s'unissant, donnèrent naissance à Éther et à Héméra, le jour ${ }^{2}$. Par le mot Éther, les Grecs entendaient les Cieux, distingués des corps lumineux ${ }^{3}$, mais par extension il représente l'espace même, le territoire situé entre les astres, un espace vide qu'il faut remplir par le souffle humain, un souffle créateur. Du côté du personnage bazinien, l'Éther est perçu comme un macro-univers au niveau duquel se construit l'existence humaine même, mais, d'autre part, il est vu aussi comme une présence qui se veut transgressée par le penchant pour le mouvement d'un héros hanté par un " éternel repart ${ }^{4}$, un goût protéique pour la transformation, l'innovation et l'adaptation à son propre style.

Tout d'abord, en tant que quatrième élément de l'univers, l'air est mis en évidence dans le roman bazinien par le goût du voyage chez le héros. Ainsi, il se lance dans une expédition constante avec pour but de transférer ses pulsions intérieures à l'espace en remplissant l'Éther, le vide inactif, réceptif et vulnérable à la fois. Gouverné par ce penchant, Hervé Bazin crée des personnages dominés par l'éternel repart, entraînés dans deux types de voyages complémentaires dans leur unité : le voyage aquatique, pour lequel le héros bazinien s'embarque avec la même exaltation que l'auteur pour l'espace dominé par l'eau, et le voyage terrestre qui est, sans doute, le meilleur exutoire de son réel subversif.

Dans les grands mythes fondateurs de l'humanité, le voyage a des valeurs initiatiques. Ainsi, l'Ancien Testament connaît plusieurs types de voyageurs : de la foi (Abraham), de la vérité (Jacob), de la providence (Joseph) ou de la

\footnotetext{
${ }^{2}$ F. Guirand, Mythologie générale, Paris, Larousse, 1935, p. 77.

3 P. Commelin, Mythologie grecque et romaine, Paris, Bordas, 1991, s. v. "L'Érèbe ", p. 3.

4 Puisque le penchant du personnage d'Hervé Bazin ne consiste pas à revenir vers les origines, mais à s'en éloigner le plus possible, nous comprenons ici par " éternel repart » une reprise bazinienne du concept nietzschéen de l’" éternel départ ».
} 
fidélité (Moïse) ${ }^{5}$. D'autre part, le Nouveau Testament ouvre la voie d'un voyage d'apprentissage, entamé par les apôtres qui accompagnent Jésus-Christ dans sa mission d'évangélisation. Du côté de la mythologie, il convient de souligner la présence d'Homère avec son Odyssée, récit qui présente le grand voyage initiatique d'Ulysse, dans son expédition de retour après la guerre de Troie. À ce propos, Arnold Van Gennep, l'ethnologue allemand, affirme : "Quant aux rites du retour du voyageur, ils comportent des rites d'enlèvement des impuretés contractées en voyage (séparation) et des rites d'agrégation progressive ${ }^{6}$. Ainsi, afin d'atteindre la purification totale, Ulysse doit entreprendre ce voyage d'une durée de vingt ans et franchir toutes les étapes de son initiation : par la suite, si le premier grand voyage à faire est au royaume de Hadès, aux Enfers, il fait équivaloir la vie à un long voyage aboutissant au même dénominateur commun - la mort.

La littérature s'empare de cette thématique si vaste et largement exploitée dans les écrits de tous les siècles: suite à son voyage en Europe qu'il entreprend afin de chasser la mélancolie, Montaigne écrit un Journal de voyage $^{7}$, découvert et publié de façon posthume. Plus tard, intéressé par l'Italie, Sade rédige son Voyage à Naples. Au XIX siècle, Théophile Gautier écrit le Voyage en Espagne et Nerval publie son récit autobiographique Voyage en Orient. Grand passionné de l'Orient, Flaubert entame lui aussi un voyage en compagnie de son ami Maxime du Camp, à la suite duquel il publiera son Voyage en Orient. Le siècle tumultueux de Proust ouvre la voie à André Gide qui publie en 1927 le récit de son Voyage au Congo. Mais si les grandes personnalités de l'art romanesque peignent des paysages telluriques, dans la

5 J. Chesseron, Voyages - Ancien Testament, https://www.eglise-niort.net/ Voyages-Ancien-Testament.

${ }^{6}$ A. van Gennep, Les rites de passage, Paris, Librairie Stock, 1924, p. 46.

${ }^{7}$ M. de Montaigne, Journal de voyage, Paris, Gallimard, 1983. 
poésie, par contre, le thème du voyage renvoie, le plus souvent, à l'univers marin : citons, ainsi, Le bateau ivre de Rimbaud ou le recueil Alcools d'Apollinaire, des arts poétiques ciblés sur le mythe du voyage initiatique dans la poésie.

Pareillement, chez Hervé Bazin aussi apparaît cette vénération de l'espace, englobant des connotations positives et négatives à la fois : si Jean, protagoniste de $L a$ Mort du petit cheval ${ }^{8}$ choisit une sortie en amoureux avec Monique en bateau sur la Marne, les trois frères Rezeau du dernier roman de la trilogie se promènent en barque sur l'Ommée afin de revivre un instant de leur enfance et l'inconnu de L'Église verte accomplit la dernière phase de son initiation en sauvant la tante Mélanie du déluge. Cependant, il y a aussi des franchissements interdits, porteurs de deuil (dans Qui j'ose aimer) ou de désolation (Les Bienheureux de La Désolation) : Belle transgresse I'Erdre afin de rentrer chez elle avec son nouveau mari elle sera punie par un lupus inguérissable. D'autre part, les habitants de Tristan traversent l'Atlantique afin de quitter l'île ou de rentrer chez eux - mais, puisque leur voyage est forcé par les circonstances, ils ne sont que des Joseph de l'Ancien Testament, victimes de leur propre destin.

En revanche, le voyage le plus chargé de sens reste, chez Hervé Bazin, le voyage tellurique, emblème du besoin effréné de mouvement et preuve d'une envie délirante de chasser ses fantasmes et d'accéder à l'Éther. Si Montaigne entame un voyage en Europe afin de guérir sa mélancolie,

\footnotetext{
${ }^{8} \mathrm{H}$. Bazin, La Mort du petit cheval, Paris, Grasset, 1950. Dorénavant, vu que le corpus de ma recherche renvoie à un grand nombre de textes, les citations renvoyant aux romans baziniens seront notées entre parenthèses avec la seule mention des initiales, comme ce qui suit : Vipère au poing (VAP), La Tête contre les murs (TCM), La Mort du petit cheval (MPC), Lève-toi et marche (LTEM), Qui j'ose aimer (QJA), Au Nom du fils (ANDF), L'Huile sur le feu (HSF), Le Matrimoine (LM), Les Bienheureux de la désolation $(B L D)$, Cri de la chouette $(C D C)$, L'Église verte (EV), Le Démon de minuit $(D D M)$ et $(N J)$ pour Le Neuvième jour. Les éditions pour chaque roman sont en concordance avec la bibliographie finale.
} 
Hervé Bazin s'attaque à un changement perpétuel du domaine qu'il choisit d'habiter. En 1977, après la publication de Ce que je crois, Hervé Bazin présente sa nouvelle demeure, en affirmant: " Je l'ai achetée il y a près de cinq ans, pour le prix d'un cinq pièces à Paris. Mais c'était une ruine. Je suis d'ailleurs un spécialiste de la ruine. Avec ma femme Monique, nous avons tout refait nous-mêmes. [...] Nous avons tout refait dans chacune de nos maisons $"{ }^{9}$. D'ailleurs, pour ainsi éviter l'étiolement romanesque, l'écrivain même explique ce penchant pour le changement, qui serait dû, selon lui, au refus de prendre racine : "Lorsque j'ai écrit trois ou quatre romans dans le même cadre, j'éprouve le besoin d'en changer. Et chaque fois que je déménage, j'ai l'impression de me renouveler ${ }^{10}$. Cependant, se déplacer constamment dans l'Éther semble ne pas soulager l'écrivain si ce n'est que pour manier sa plume; dès qu'elle sèche, il repart à la recherche de l'encre tutélaire, indispensable à la survie de l'auteur et de l'écriture même ${ }^{11}$.

C'est ainsi que, loin d'être un simple passe-temps, ce " vice d'architecte " aurait, en fin de compte, un but cathartique, des propriétés guérissantes : " J'ai habité une douzaine de maisons différentes après avoir vendu la propriété familiale pour des raisons sentimentales ${ }^{12}$,

${ }^{9}$ H. Bazin, "Hervé Bazin : il a choisi le retour à la terre », reportage de M. F. Saurat, [dans :] Paris Match, 1977, n 1455, p. 35.

10 Ibidem.

${ }^{11}$ Son espace privilégié est la France en général et le Pays de la Loire en particulier. À ce propos, Jean Anglade affirme : " après deux séjours en Canada, Hervé Bazin revient déçu et vide de sujet " (J. Anglade, Hervé Bazin, Paris, Gallimard, 1962, p. 64). Après de nombreux voyages en Amérique, en Afrique ou en Europe, il se rend compte que le pays étranger ne réveille pas son souffle créateur, justement parce que ce n'est pas le sien. Anglade souligne encore la révolte de l'écrivain contre les langues étrangères même, en citant Hervé Bazin : " J'ai trop le souci de cultiver ma propre langue pour pouvoir m'occuper d'une autre " (H. Bazin cité d'après : J. Anglade, Hervé Bazin, op. cit., p. 65).

$12 \mathrm{H}$. Bazin, "Interview de Hervé Bazin ", dans une interview accordée à Major Serge Voeltzel, [dans :] L'étincelle, 1984, n 174, p. 36. 
constate l'écrivain en 1984, histoire présentée une trentaine d'années auparavant lors de la publication de son roman La Mort du petit cheval : le protagoniste de I'histoire, alter ego de l'écrivain, rachète la maison familiale à son frère pour la revendre en petits lots peu de temps après. C'est ce que son personnage Pascal, de Lève-toi et marche, choisira de faire, lui aussi, après la mort de sa mère, geste qu'il explique brièvement :

Je vais vendre la maison de ma mère. Dans mon état, il n'est pas souhaitable d'être retenu par des biens qui ont des racines en terre, qui fixent un homme dans le souvenir et par là même peuvent arriver à localiser son avenir, à le réduire dans l'espace. Je vends la maison de ma mère, précisément parce que je sens que j'y tiens. (LTEM, 192)

Ainsi, il ne faut pas être fixé dans le souvenir de la maison d'enfance : et alors, si Pascal ressent cette appartenance à l'espace, il essayera aussi de la chasser par la raison et la combattre par le geste. II le fera au nom de cet éternel repart imposé par sa religion, qui l'oblige à couper les racines de cet arbre qui grandit et dont l'origine pourrait gâcher sa mission dans le monde. Lui, il est censé suivre l'exemple de Jésus proclamant aux apôtres : " Les renards ont des tanières, et les oiseaux du ciel ont des nids : mais le Fils de l'Homme n'a pas de lieu où il puisse reposer sa tête $»^{13}$.

Le souci de ne jamais trop se fixer, en gardant pour toute sa vie la vertu de restauration, définit la vision d'Hervé Bazin sur les demeures, qu'il expose généreusement dans son Abécédaire : "Virtuose des domiciles, j'habite ; je ne demeure guère ${ }^{114}$, constate l'écrivain. En 1972, guidé par le même sentiment, le philosophe Jacques Rolland de Renéville écrivait : " Envisagé comme impossibilité d'être partout mais comme liberté d'être

${ }^{13}$ La Bible de Jérusalem, traduite en français sous la direction de l'École biblique de Jérusalem, Paris, Éditions du Cerf, 2000, Luc $9: 58$.

14 H. Bazin, Abécédaire, Paris, Grasset \& Fasquelle, 1984, s. v. « Demeures », p. 77. 
toujours ailleurs, à condition de ne jamais se fixer, l'espace apparaît la monotone pauvreté d'une répétition infinie qui ne produit ou même ne reproduit jamais rien. Plutôt ici qu'ailleurs, puisque, à défaut d'être partout, exister sera toujours n'être que là, puis un peu plus loin, et enfin n'être nulle part ? ${ }^{15}$.

L'espace est construit pour être habité et afin de chasser la monotonie, il est voué à la diversité : "J'ai occupé successivement une quinzaine de maisons, jamais en ville [...]. Et le souci de ne jamais trop me fixer ${ }^{16}$. Hervé Bazin construit pour vivre et non pour y vivre, il revigore ses demeures, les remet à la vie et une fois son objectif atteint, il s'en désintéresse : "Posséder, ne m'intéresse guère. J'ai le vice de l'architecte, qui construit ou transforme, puis se désintéresse de ce qu'il a achevé ${ }^{17}$, remarque l'écrivain. Et puisque pour Hervé Bazin " l'être est supérieur à l'avoir $\|^{18}$, ce goût de la privation du matériel habite aussi la pensée des personnages qu'il entame : "Dans succéder il y a céder » $(N J, 35)$, songe le protagoniste du Neuvième jour.

Son personnage inconnu de L'Église verte est décrit, lui aussi, comme indépendant des biens matériels. Comme l'avoue l'étranger :

Sommés de consommer, vous êtes accomplis, vous êtes possédés par ce qui vous appartient. Moi, c'est le luxe inverse qui m'intéresse : vivre sans biens, sans règle, sans sécurité, sans ambition, sans mémoire, sans nom... $(E V, 211)$,

15 J. R. de Renéville, Aventure de l'absolu, cité d'après : J.-Y. Tadié, Le récit poétique, Paris, Gallimard, 1994, p. 57.

16 J. Boislève, "Notes en marge de l'Abécédaire ", [dans :] A.-S. Dufief (dir.), Hervé Bazin - connu et inconnu, Actes du colloque des 14-15 décembre 2007 tenu à Angers et Fontevraud, Angers, Éditions Presse de I'Université d'Angers, 2009, p. 30.

17 J.-C. Lamy, Hervé Bazin. Entretiens avec Jean-Claude Lamy, Paris, Stock, 1992, p. 53.

${ }^{18}$ F. Brengues-Épinette, «Faire, défaire, refaire chez Hervé Bazin », [dans :] Hervé Bazin : actes du colloque d'Angers du 11 au 13 décembre 1986, Centre de recherches en littérature et linguistique de l'Anjou et des Bocages, Angers, Presses de l'Université d'Angers, 1987, p. 293. 
Curieusement, dans ce roman, l'alter ego de l'écrivain n'est pas le protagoniste-narrateur - Jean-Luc Godion mais précisément cet inconnu, dont la voix semble résonner comme un tonnerre, rappelant la grandeur divine, la création, l'écrivain-même : "Ne rien dire, ne rien être, ne rien avoir, c'est toute son ambition » $(E V, 161)$.

Très lié d'amitié avec Grasset, dont la maison d'édition a publié la plupart de ses romans, Hervé Bazin le cite dans l'une de ses interviews : "J'ai honte de posséder, ce que je veux c'est donner ma forme ${ }^{19}{ }^{19}$, soutenait le Père Grasset, logeant dans un hôtel et ne possédant que la maison d'édition. Pareillement, dans le même entretien, Bazin constate : "J'ai toujours été un peu honteux de posséder $\aleph^{20}$. De ce fait, à supposer que pour Pascal (LTEM), la vente de la maison familiale soit une pierre de touche, une étape incontournable de son initiation, par extension, la plupart des personnages baziniens regardent le mouvement comme un principe gouvernant leur existence. C'est ainsi que, lors d'une interview accordée à Jean-Claude Lamy, Bazin constate : " J'ai dû faire une allergie aux murs. En tout cas, la stabilité n'aura pas été mon point fort ${ }^{21}$.

$\mathrm{Si}$, pour l'écrivain, déménager signifie raréfier l'oxygène, chasser d'autres sources d'inspiration, pour le héros bazinien le mouvement est la sève de la vie même. Le penchant pour la création de personnages gouvernés par la bougeotte, cette folie qui empêche l'individu de trouver sa sérénité, entraîne un bon nombre de personnages baziniens : " À vrai dire mon bonheur serait volontiers ambulatoire, du moins amateur de changement, de dépaysement » $(L M, 227)$, écrit Abel Bretaudeau. Le concept de déterritorialisation, propre à Derrida, s'applique

${ }^{19}$ H. Bazin, Lhoste P., une interview enregistrée en 1969, distributeur : Institut national de l'audiovisuel, consultée à la Bibliothèque Nationale de France, cote : PDC12-1642.

20 Ibidem.

21 J.-C. Lamy, Hervé Bazin. Entretiens avec Jean-Claude Lamy, op. cit., p. 53. 
admirablement au caractère des personnages baziniens, l'écrivain même étant une victime de la reterritorialisation. Prenons un premier exemple : Brasse-Bouillon - le premier aventurier de l'œuvre bazinienne. Fabienne Épinette ${ }^{22}$ distingue dans son étude trois types de voyages dans Vipère au poing seulement : un premier, de la BelleAngerie à Angers afin de visiter Folcoche à la clinique, voyage qui semble insignifiant aux yeux du jeune protagoniste ; un deuxième, en compagnie de son père et de ses deux frères d'Angers jusqu'à Armagnac et un troisième, précurseur des révoltes adolescentes : le voyage à Paris. Si, par sa biographie, Hervé Bazin paraît être un grand voyageur, son alter ego est un " être mobile ${ }^{23}$ par ses nombreuses escapades de la maison et par le refuge qu'il cherche au sommet de la maison; comme l'affirme le protagoniste de Vipère au poing :

Je ne sais pas pourquoi, mais, perché tout là-haut, je me sens tout autre. Dominant les toits bleus de La Belle-Angerie et uniquement dominé par le vent d'ouest ou les ramiers qui tournent longuement autour de leurs nids, je me détache de ma vie. (VAP, 228)

L'air est l'issue de secours de Brasse-Bouillon, qui se détache d'une vie pleine de contraintes imposées par une mère autoritaire. De ce fait, il y $a$, dans ses mots, un désir latent de dominer le monde, réussi par le personnage en montant sur les toits de la Belle-Angerie ou au plus haut du taxaudier, et par l'écrivain à travers l'écriture, élevée au rang le plus sublime de l'art poétique. Ainsi, la chasteté du corps et de l'esprit que ses personnages recherchent à tout prix renvoie, sur le plan artistique, à la pureté de l'écriture : de ce point de vue, l'œuvre littéraire représente la quintessence, le produit le plus raffiné et filtré. Hervé Bazin entame, ainsi, une écriture qui s'oblige à être pure, travaillée, bien soignée ; comme l'affirme Guillaume

22 F. Épinette Brengues, Hervé Bazin. Vipère au poing, Paris, Ellipses Marketing, 2000, p. 45-47.

23 Ibidem, p. 46. 
Apollinaire dans son recueil Alcools. Ainsi, tout comme le vin est le produit des raisins qui supposent un certain délai pour que la germination rende la quintessence, l'œuvre littéraire demande un travail titanesque, mais qui aboutit à l'esthétique. Le roman bazinien est, conséquemment, un art poétique, une écriture produite pour satisfaire le goût pour la beauté et un artifice de grâce qui vise le sublime.

Mais l'air est aussi l'espace de la liberté des oiseaux, qui par la rêverie seule cicatrise les blessures de l'esprit : "L'aigreur de l'air, assortie à la vivacité des oiseaux, au fil coupant de l'herbe, me fit du bien " (QJA, 137), raconte Isa. En fait, chez les personnages baziniens il y a toujours un espace de refuge : si pour Isa (protagoniste de Qui j'ose aimer), Céline (L'Huile sur le feu) ou pour l'inconnu de la Lagrairie (L'Église verte) on observe la nature sauvage, pour Brasse-Bouillon c'est le sommet du taxaudier qui lui adoucit le goût de liberté grâce à " l'air pur " (VAP, 137) qu'il y ressent. Symboliquement associé au vent et au souffle ${ }^{24}$, l'air représente, ainsi, la pureté, la renaissance de l'esprit, l'endroit où le moi intérieur retrouve son identité perdue.

Revenons, en ce qui suit, à l'esprit de mouvement, qui individualise l'écrivain et ses personnages, dont le premier est le jeune Brasse-Bouillon (VAP). Si Pierre Moustiers caractérise Hervé Bazin de "romancier en mouvement ${ }^{25}$ ce n'est pas par hasard et j'opine, avec Fabienne Épinette, que son premier personnage proclame déjà cette « Grande Marche ${ }^{26}$ du début à la fin du roman : "Je suis celui qui marche, une vipère au poing " (VAP, 256), avoue le protagoniste de Vipère au poing.

Un autre héros construit en mouvement est Constantin ler - le personnage historique du roman de Gérard

24 J. Chevalier, Dictionnaire des synonymes, Paris, Robert Laffont, 1969, s. v. " Air », p. 19.

25 P. Moustiers, Hervé Bazin ou le romancier en mouvement, Paris, Seuil, 1973.

${ }^{26}$ F. Épinette Brengues, Hervé Bazin. Vipère au poing, op. cit., p. 46. 
Laguenière $(D D M)$. Afin de prouver sa force et pour agrandir son empire, il mène une vie en mouvement, sur le champ de bataille : lui, il est un combattant et s'il bâtit sa maison sur le front, il le fait en vue de conquérir le pouvoir. De l'autre côté se trouve Gérard qui, dans le processus de création de son personnage, passe par des rêveries historiques et par des voyages initiatiques rimbaldiens : dans son Bateau ivre symbolique, il transgresse la frontière de la France afin de retrouver sa maîtresse wallonne.

Mais le personnage le plus hanté par ce penchant pour le mouvement reste Arthur Gérane (protagoniste de La Tête contre les murs) qui, lors d'une escapade de l'asile de Vaucluse, s'engage dans un périple continu afin de guérir sa frénésie : la Côte d'Azur, la Corse, Cannes ou les Vosges sont ses principales attractions. C'est notamment dans le mouvement qu'il trouve la stabilité, dans le périple continu, un éternel départ. Arthur ne s'arrête pas au cours de son voyage, tout comme son créateur au cours de l'écriture :

Ainsi commençait le plus inutile, le plus absurde périple. Banal ou pittoresque, l'horizon n'avait aux yeux d'Arthur qu'une seule vertu : celle du changement. II ne savait pas voyager : il se déplaçait. II obéissait, dans l'état de mouvement, à la loi d'inertie. Pas le moindre goût de la découverte, qui fait l'explorateur, ni de la flânerie, qui fait le touriste ; mais celui de la bougeotte. Une seule province d'élection : celle où il n'était pas. (TCM, 232)

Le voyage, chez Hervé Bazin, ne se fait pas dans des buts extérieurs, culturels ou artistiques, mais pour le simple plaisir de ne pas rester en place, de bouger tout le temps. "Voyager, c'est guérir son âme "27, constate Michel Deguy - philosophe, poète et essayiste contemporain. Selon le même principe, le personnage bazinien se trouve dans une permanente quête identitaire, une recherche de stabilité intérieure qu'il ne peut trouver que

${ }^{27}$ M. Deguy, La raison poétique, Paris, Galilée, 2000, p. 41. 
dans la bougeotte. II ne peut apaiser sa révolte que dans l'inutilité apparente et incontournable à la fois, dans ce périple qui offre au lecteur une description minutieuse des endroits visités et, en même temps, une peinture des maisons de santé. Pour quelques mois, il s'éprend très vite de la vie campagnarde, là où sa folie devient inaperçue, là où son inquiétude diminue. Pendant les huit mois qu'il passe dans la ferme d'un paysan, il est vu comme un bon travailleur, sain et responsable, mais la peur de l'engagement et de la stabilité le fait renoncer à tout : famille, santé et sérénité.

Et comme l'errance présente les meilleures occasions pour les plus éblouissantes rencontres, Arthur s'attache à un bonheur passager qui survient dans sa vie : il rencontre Stéphanie, la plus belle fille d'un village au cœur des Faucilles, au fil de la Saône, dans les Vosges. Il s'y installe par hasard, suite à une panne de voiture et arrive à épouser la jeune Wallonne ; c'est ici qu'il découvrira la santé de l'esprit et finira par se retrouver lui-même à l'aide de la nature qui l'abrite : "Depuis que Stéphanie connaissait Arthur, celui-ci s'était toujours montré calme, rangé, sobre, travailleur " (TCM, 255). Les villageois le confirment : "Ceux qui prétendent qu'Arthur est fou, ditil, ce sont eux qui ont l'esprit dérangé. Il a vécu avec nous pendant des mois : nous nous en serions bien aperçus. II n'y a pas plus calme que ce garçon-là. Je ne l'ai même pas vu une seule fois éméché » (TCM, 258).

Pendant les huit mois qu'il passe au milieu des montagnes, il s'attache au silence de l'endroit et à celui de sa femme : "Tout de suite, Arthur fut impressionné par son silence " (TCM, 259). Réenfermé, il s'évade de l'asile et s'attaque à un nouveau périple en quête du silence guérissant et de la solitude qui ne lui sera pas réservée. Atout préféré de Folcoche (VAP), le silence est un leitmotiv du roman bazinien, dont l'apogée est constitué par L'Église verte, histoire inspirée d'un fait divers, où l'écrivain donne vie à cet inconnu, découvert dans la forêt : 
ici, les personnages vivent en accord avec la nature, la vraie protagoniste du roman, et lui confèrent le statut d'instance suprême. Le texte fait du silence une thématique récurrente, puisqu'il est le principe qui gouverne un bon nombre de personnages de ce roman et pas seulement. Léonard, filleul des Godion, est un introverti : "Parlant peu, Léonard supporte bien le silence " $(E V, 79)$, Laure $(A N D F)$, la bonne tante qui se charge d'élever ses neveux, est une "silencieuse " $(A N D F, 67)$. Bertrand Colu (HSF) en a, lui aussi, une grande dévotion : "Le silence est d'une qualité rare, il refuse le bruit, et nos pas n'y peuvent rien "(HSF, 194). Mais c'est l'inconnu, Hix, Mutix ou M. Trente de L'Église verte qui se situe, par contre, au point culminant.

Finalement, parmi les quatre éléments primordiaux, l'air est, pour le personnage bazinien, le principe de (sur)vie. Afin de vider son esprit, déjà trop habité par l'espace qu'il vit, Hervé Bazin recourt au baptême de l'air, qu'il confère aussi à ses personnages. Ainsi, lors de son premier grand voyage, une visite aux Canaries avec Mme Rezeau sa grand-mère, Salomé affirme : " C'est mon baptême de l'air " $(C D C, 141)$. Mais le baptême porte ici une différence par rapport au baptême de l'eau : à chaque surabondance de l'Éther, l'espace est censé être changé. C'est ainsi que pour chaque ruine qu'il a provoquée lui-même, Hervé Bazin propose une (re)construction, ce qui, en outre, l'intéresse le plus : "Dans posséder, j'entends c é de $r$ et je suis ravi de ce qui me reste $1{ }^{28}$. Finalement, on peut dire qu'il habite et est habité en même temps par l'espace, qu'il doit diversifier pour survivre et pour créer.

En 1926, Chklovski écrivait : " L'œuvre est entièrement construite, toute sa matière est organisée ${ }^{29}$. En plein

${ }^{28}$ H. Bazin, Ce que je crois, Paris, Bernard Grasset, 1977, p. 80.

29 V. Chklovski cité par T. Todorov, Poétique de la prose, Paris, Seuil, 1971, p. 12. 
formalisme russe, la théorie de Jakobson, qui mise sur la littérarité de l'œuvre, se développe au jour le jour et l'essence de l'œuvre séjournera, dorénavant, dans le procédé, le comment de l'écriture qui rattache l'écrit à l'art. Ainsi, dans son crédo artistique, gourmand de l'action comprise dans la démolition et, à la fois, dans la création, Hervé Bazin deviendra l'écrivain du verbe, de l'initiative et de la démolition. Concluons avec Jean Anglade, qui définit cette passion de l'auteur comme un sixième sens : "La construction chez lui n'est pas une manie, mais un sixième sens. Un sens hypertrophié. Hervé Bazin est glouton de la construction ${ }^{30}$. L'écrivain même griffonne son crédo artistique en quelques mots, en affirmant : " Du roman qui détruit, j'ai voulu passer au roman constructeur $»^{31}$.

30 J. Anglade, Hervé Bazin, op. cit., p. 12.

${ }^{31} \mathrm{H}$. Bazin dans une interview accordée à Gabriel Aubarède, [dans :] Les nouvelles littéraires du 19 juin 1952, nº de page inconnu; consulté à la Bibliothèque Universitaire d'Angers, au fonds « Hervé Bazin », R740433. 


\section{bibliographie}

Anglade J., Hervé Bazin, Paris, Gallimard, 1962.

Bazin H., Abécédaire, Paris, Grasset et Fasquelle, 1984.

Bazin H., Au Nom du fils, Paris, Seuil, 1960.

Bazin H., Ce que je crois, Paris, Grasset, 1977.

Bazin H., Cri de la chouette, Paris, Grasset, 1972.

Bazin H., Voeltzel S., "Interview de Hervé Bazin ", [dans :] L'étincelle, $1984, \mathrm{n}^{\circ} 174$.

Bazin H., L'Église verte, Paris, Seuil, 1981.

Bazin H., L'Huile sur le feu, Paris, Grasset, 1954.

Bazin H., La Mort du petit cheval, Paris, Grasset, 1950.

Bazin H., La Tête contre les murs, Paris, Grasset, 1949.

Bazin H., Le Démon de minuit, Paris, Grasset, 1988.

Bazin H., Le Matrimoine, Paris, Seuil, 1967.

Bazin H., Le Neuvième jour, Paris, Grasset, 1994.

Bazin H., Les Bienheureux de la désolation, Paris, Seuil, 1970.

Bazin H., Lève-toi et marche, Paris, Grasset, 1952.

Bazin H., Qui j'ose aimer, Paris, Grasset, 1956.

Bazin H., Vipère au poing, Paris, Grasset, 1948.

Bazin H., Lhoste P., une interview enregistrée en 1969, distributeur : Institut national de l'audiovisuel, consultée à la Bibliothèque Nationale de France, cote : PDC12-1642.

Bazin H., une interview accordée à Gabriel Aubarède, [dans :] Les nouvelles littéraires du 19 juin 1952, nº de page inconnu ; consulté à la Bibliothèque Universitaire d'Angers, au fonds " Hervé Bazin », R740433.

La Bible de Jérusalem, traduite en français sous la direction de l'École biblique de Jérusalem, Paris, Éditions du Cerf, 2000.

Boislève J., "Notes en marge de l'Abécédaire », [dans :] A.-S. Dufief (dir.), Hervé Bazin. Connu \& inconnu, actes du colloque des 14-15 décembre 2007 tenu à Angers et Fontevraud, Angers, Presses de l'Université d'Angers, 2009.

Brengues-Épinette F., "Faire, défaire, refaire chez Hervé Bazin », [dans :] Hervé Bazin : actes du colloque d'Angers du 11 au 13 décembre 1986, Centre de recherches en littérature et linguistique de I'Anjou et des Bocages, Angers, Presses de l'Université d'Angers, 1987.

Brengues-Épinette F., Hervé Bazin. Vipère au poing, Paris, Ellipses Marketing, 2000.

Chesseron J., Voyages - Ancien Testament, https://www.eglise-niort.net/ Voyages-Ancien-Testament.

Chevalier J., Dictionnaire des synonymes, Paris, Robert Laffont, 1969.

Commelin P., Mythologie grecque et romaine, Paris, Bordas, 1991.

Deguy M., La raison poétique, Paris, Galilée, 2000.

van Gennep A., Les rites de passage, Paris, Librairie Stock, 1924.

Guirand F., Mythologie générale, Paris, Larousse, 1935.

Haraucourt E., Seul, Paris, Bibliothèque Charpentier, 1891.

Lamy J.-C., Hervé Bazin. Entretiens avec Jean-Claude Lamy, Stock, Paris, 1992.

Montaigne M. de, Journal de voyage, Paris, Gallimard, 1983. 
Moustiers P., Hervé Bazin ou le romancier en mouvement, Paris, Seuil, 1973.

Saurat M. F., " Hervé Bazin : il a choisi le retour à la terre ", [dans :] Paris Match, 1977, n० 1455.

Tadié J.-Y., Le récit poétique, Paris, Gallimard, 1994.

Todorov T., Poétique de la prose, Paris, Seuil, 1971.

\section{abstract}

Hervé Bazin and the air baptism - gratification of the God Ether

The aim of this paper is to bring into discussion the work of Hervé Bazin, a great novelist of the $X X^{\text {st }}$ century, drawing on the concept of travelling in his novels. Preferring the telluric universe instead of the river, Hervé Bazin transforms the scene of his novel into a privileged space for writing. And as the writing is original, it demands a continuous resumption and a wide area of each published novel: in this way, the Bazinian character is defined by a spirit of wanderling, being fascinated by Ether, this empty space that he fills in with his creative mind and with the dynamism of his self.

\section{keywords}

travelling, Ether, movement, writing

\section{mots-clés}

voyage, Éther, bougeotte, écriture

\section{bianca-livia bartoș}

Bianca-Livia Bartoș est doctorante à la Faculté des Lettres de l'Université Babeş-Bolyai et professeur de FLE. Sous la direction de Madame le Professeur Yvonne Goga, elle rédige une thèse qui porte le titre Avatars d'une écriture poétique chez Hervé Bazin. D'ailleurs, la thématique de l'œuvre bazinienne se trouve au centre de ses intérêts depuis son mémoire de dissertation intitulé Hervé Bazin : de l'humour à l'art poétique. 